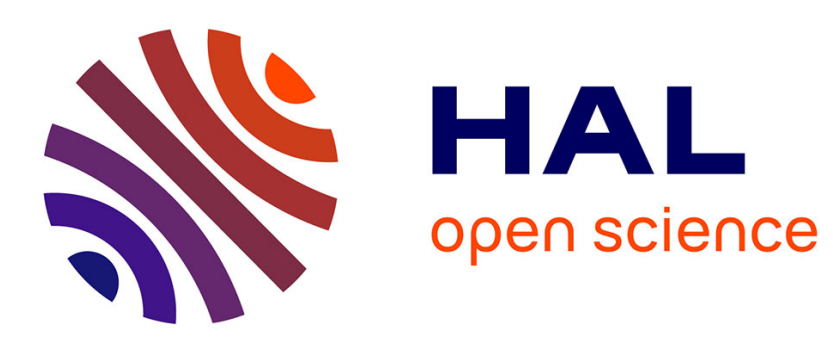

\title{
2D $1 / 2$ visual servoing stability analysis with respect to camera calibration errors
}

E. Malis, François Chaumette, S. Boudet

\section{To cite this version:}

E. Malis, François Chaumette, S. Boudet. 2D 1/2 visual servoing stability analysis with respect to camera calibration errors. IEEE/RSJ Int. Conf. on Intelligent Robots and Systems, IROS'98, 1998, Victoria, Canada, Canada. pp.691-697. inria-00352555

\section{HAL Id: inria-00352555 \\ https://hal.inria.fr/inria-00352555}

Submitted on 13 Jan 2009

HAL is a multi-disciplinary open access archive for the deposit and dissemination of scientific research documents, whether they are published or not. The documents may come from teaching and research institutions in France or abroad, or from public or private research centers.
L'archive ouverte pluridisciplinaire HAL, est destinée au dépôt et à la diffusion de documents scientifiques de niveau recherche, publiés ou non, émanant des établissements d'enseignement et de recherche français ou étrangers, des laboratoires publics ou privés. 


\title{
$2 \mathrm{D} 1 / 2$ visual servoing stability analysis with respect to camera calibration errors
}

\author{
Ezio Malis \\ IRISA/INRIA \\ Campus de Beaulieu, \\ François Chaumette \\ IRISA/INRIA \\ Campus de Beaulieu, \\ Sylvie Boudet \\ 35042 Rennes, France. \\ 35042 Rennes, France. \\ EDF/DER Chatou \\ 6 Quai Watier, \\ 78401 Chatou, France.
}

\begin{abstract}
In this paper, the robustness of a new visual servoing scheme with respect to camera calibration errors is analyzed. This scheme, called 2D 1/2 visual servoing, is based on the estimation of the partial camera displacement from the current to the desired camera poses at each iteration of the control law. Visual features and data extracted from the partial displacement allow us to design a decoupled control law controlling the six camera d.o.f. The necessary and sufficient conditions for local asymptotic stability in presence of camera calibration errors are easily obtained. Then, thanks to the simple structure of the system, sufficient conditions for global asymptotic stability are proposed. Finally, experimental results show the validity of our approach and its robustness not only with respect to camera calibration errors but also to robot calibration errors.
\end{abstract}

\section{Introduction}

Vision feedback control loop was introduced in order to increase the flexibility and the accuracy of robot systems [6, 7]. Consider for example the classical positioning task of an eye-in-hand system with respect to a target. After the image corresponding to the camera desired position has been learned, and after the camera and/or the target has been moved, an error control vector can be extracted from the two views of the target. A zero error implies that the robot end-effector has reached the desired position with an accuracy regardless to calibration errors. However, these errors influence the way the system converges. In many cases image features may get out of the camera field of view during the servoing, which thus lead to its failure. For this reason, it is important to study the visual servoing robustness with respect to calibration errors.

Position-based visual servoing necessitates a calibrated camera and the knowledge of a perfect geometric model of the target to obtain unbiased pose esti- mation from image features [11]. Even if a closed loop control is used, which makes possible the convergence of the system in presence of calibration errors, it is quite impossible to analytically analyze the stability of the system. Image-based visual servoing is known to be robust with respect to calibration errors [3]. However, its convergence is theoretically ensured only in a region (quite impossible to determine analytically) around the desired position. Except in simple cases, the analysis of the stability robustness with respect to calibration errors is again quite impossible, since the system is coupled and non-linear [3].

It is shown in this paper that, contrarily to the previous approaches, it is possible to obtain analytical results using the $2 \mathrm{D} 1 / 2$ visual servoing approach [9]. 2D $1 / 2$ visual servoing is based on the projective reconstruction of the target using feature points extracted from two images (corresponding to the current and desired camera poses). Let us emphasize that this reconstruction does not necessitate the knowledge of the $3 \mathrm{D}$ position of the target points, which makes unnecessary a 3D model of the target, and increases the versatility and the application area of visual servoing.

If the camera intrinsic parameters are known, a scaled Euclidean reconstruction is obtained from the projective reconstruction, and the camera rotation between the two views is computed at each iteration. Consequently, the rotational and translational control loop can be decoupled which allows to obtain the convergence of the positioning task in all the task space. In order to control the three remaining camera d.o.f, we introduce the extended image coordinates of a reference point of the target. These ones are obtained from the classical normalized image coordinates by adding a third normalized $z$ coordinate which is measured from the Euclidean reconstruction. The interaction matrix which links the time derivative of the extended image coordinates and the camera velocity screw, called the 
extended image Jacobian matrix, has not singularity.

If the camera intrinsic parameters are not perfectly known, the estimated control vector can be analytically computed in function of camera calibration errors. Then, the necessary and sufficient conditions for the local asymptotic stability in presence of camera calibration errors are easily obtained. Moreover, thanks to the simple structure of the system, sufficient conditions for global asymptotic stability are presented.

\section{Euclidean reconstruction}

\subsection{Projective Homography estimation}

Consider three 3D target points $\mathbf{P}_{i}$ belonging to a reference plane $\pi$ (see Figure 1). It is well known that the resulting image points $\mathbf{m}_{i}$ in the current camera frame $\mathcal{F}$ (expressed in pixels), are related to the corresponding ones $\mathbf{m}_{i}^{*}$ in the desired camera frame $\mathcal{F}^{*}$, by a projective homography $\mathbf{H}_{p}$ such that $(i=1,2,3)$ $\mathbf{m}_{i}=\mathbf{H}_{p} \mathbf{m}_{i}^{*}$ [5]. If $n$ supplementary points $\mathbf{P}_{j}$ does not belong to the reference plane (i.e. if the target is not planar), the relationship between its current and desired image points $\mathbf{m}_{j}$ and $\mathbf{m}_{j}^{*}$ respectively, is no more linear in the unknown elements of $\mathbf{H}_{p}$. However, if $n \geq 5$ points are available, it is possible to estimate the homography matrix at video-rate using for example the linearized algorithm presented in [8].

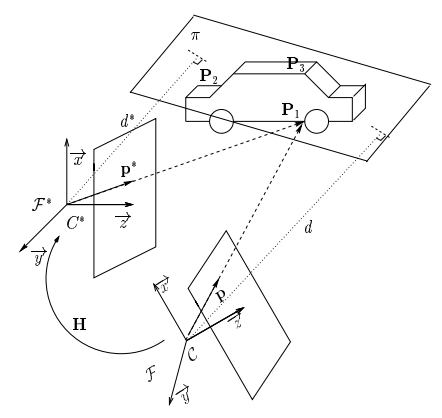

Figure 1: Camera displacement modelisation

\subsection{Known camera calibration}

Let $\mathbf{A}$ be the intrinsic parameters matrix of the camera:

$$
\mathbf{A}=\left[\begin{array}{ccc}
\alpha_{u} & \alpha_{u v} & u_{0} \\
0 & \alpha_{v} & v_{0} \\
0 & 0 & 1
\end{array}\right]
$$

This is the transformation between the pixel coordinates and the normalized coordinates of an image point. Assuming that the camera calibration is known, the Euclidean homography is calculated as follows:

$$
\mathbf{H}=\mathbf{A}^{-1} \mathbf{H}_{p} \mathbf{A}
$$

After $\mathbf{H}$ is computed, it can be decomposed as the sum of a rotation matrix and of a rank 1 matrix [5]:

$$
\mathbf{H}=\mathbf{R}+\mathbf{t}_{d^{*}} \mathbf{n}^{* T}
$$

where $\mathbf{R}$ is the rotation matrix between frames $\mathcal{F}$ and $\mathcal{F}^{*}$ (i.e. the homography of the plane at infinity $\mathbf{R}=$ $\left.\mathbf{H}_{\infty}\right), \mathbf{t}_{d^{*}}$ is the ratio between the translation vector $\mathbf{t}$ and the distance $d^{*}$ of $C^{*}$ from $\pi$, and $\mathbf{n}^{*}$ is the unit vector normal to the plane $\pi$ expressed in $\mathcal{F}^{*}$. From $\mathbf{H}$ and the image features, it is thus possible to determine these motion parameters and the structure of the reference plane. For example, the distances $d$ and $d^{*}$ are unknown (where $d$ is the current distance between frame $\mathcal{F}$ and $\pi$ ), but the ratio $r=d / d^{*}$ can easily be estimated. Indeed, noting $\mathbf{n}=\mathbf{R} \mathbf{n}^{*}$ the vector normal to $\pi$, expressed in $\mathcal{F}$, we have:

$$
r=\frac{d}{d^{*}}=1+\mathbf{n}^{T} \mathbf{t}_{d^{*}}=\operatorname{det}(\mathbf{H})
$$

Furthermore, the ratio $\rho$ between the unknown depth $Z$ of a point lying on $\pi$ and $d^{*}$, can be computed as:

$$
\rho=\frac{Z}{d^{*}}=\frac{r}{\mathbf{n}^{T} \mathbf{p}}
$$

These parameters are important since they are used in the design of our control scheme. In the following subsection we will show how it is possible, in the uncalibrated domain, to obtain an analytical form of the estimated motion parameters as a function of the real motion parameters and of the camera calibration errors.

\subsection{Unknown camera calibration}

If the camera is not perfectly calibrated and $\widehat{\mathbf{A}}$ is used instead of $\mathbf{A}$, the measured normalized image point $\widehat{\mathbf{p}}$ can be written in function of the real normalized one $\mathbf{p}$ as:

$$
\widehat{\mathbf{p}}=\delta \mathbf{A p}
$$

where $\delta \mathbf{A}=\widehat{\mathbf{A}}^{-1} \mathbf{A}$. Then, in presence of calibration errors, the estimated homography matrix is:

$$
\widehat{\mathbf{H}}=\delta \mathbf{A} \mathbf{H} \delta \mathbf{A}^{-1}
$$

The estimated homography matrix can be decomposed as the sum of a matrix similar to a rotation matrix and of a rank 1 matrix:

$$
\widehat{\mathbf{H}}=\widehat{\mathbf{H}}_{\infty}+\widehat{\mathbf{t}}_{d^{*}} \widehat{\mathbf{n}}^{* T}
$$

where $\widehat{\mathbf{H}}_{\infty}=\delta \mathbf{A} \mathbf{R} \delta \mathbf{A}^{-1}, \widehat{\mathbf{t}}_{d^{*}}=\left\|\mathbf{n}^{* T} \delta \mathbf{A}^{-1}\right\| \delta \mathbf{A} \mathbf{t}_{d^{*}}$ and $\widehat{\mathbf{n}}^{* T}=\frac{\mathbf{n}^{* T} \delta \mathbf{A}^{-1}}{\left\|\mathbf{n}^{* T} \delta \mathbf{A}^{-1}\right\|}$. The eigenvalues of $\mathbf{R}$ depend on 
the angle of rotation $\theta$, and its eigenvector corresponding to the unitary eigenvalues is the axis of rotation $\mathbf{u}$. Matrix $\widehat{\mathbf{H}}_{\infty}$ is not a rotation matrix, but is similar to $\mathbf{R}$, which implies that the two matrices have the same eigenvalues and the eigenvectors of $\widehat{\mathbf{H}}_{\infty}$ are the eigenvectors of $\mathbf{R}$ multiplied by matrix $\delta \mathbf{A}$. The estimated rotation angle $\widehat{\theta}$ and the estimated rotation axis $\widehat{\mathbf{u}}$, extracted directly from $\widehat{\mathbf{H}}_{\infty}$, can be written as a function of the real parameters and of the calibration errors:

$$
\widehat{\theta}=\theta \quad \text { and } \quad \widehat{\mathbf{u}}=\frac{\delta \mathbf{A u}}{\|\delta \mathbf{A} \mathbf{u}\|}
$$

It must be emphasized that, as well as the rotation angle $\theta$, the ratio $r$ is computed without error:

$$
\widehat{r}=\operatorname{det}(\widehat{\mathbf{H}})=\operatorname{det}(\mathbf{H})=r
$$

Consequently, since $\widehat{\mathbf{n}}=\widehat{\mathbf{R}}^{-T} \widehat{\mathbf{n}}^{*}=\frac{\delta \mathbf{A}^{-T} \mathbf{n}}{\left\|\delta \mathbf{A}^{-T} \mathbf{n}^{*}\right\|}, \widehat{\rho}$ is:

$$
\widehat{\rho}=\frac{\widehat{r}}{\widehat{\mathbf{n}}^{T} \widehat{\mathbf{p}}}=\frac{r}{\mathbf{n}^{T} \mathbf{p}}=\left\|\delta \mathbf{A}^{-T} \mathbf{n}^{*}\right\| \rho
$$

\section{$3 \quad 2 \mathrm{D} 1 / 2$ visual servoing}

In this section, we present the design of our visual servoing scheme. In order to control the orientation of the camera, we use of course the $3 \mathrm{D}$ estimated rotation $\mathbf{R}$ between $\mathcal{F}$ and $\mathcal{F}^{*}$ (which has to reach the identity matrix). Let $\mathbf{u}$ be the rotation axis and $\theta$ the rotation angle obtained from $\mathbf{R}$. The rotational velocity of the camera $\boldsymbol{\Omega}$ is simply expressed in function of the angular velocity $\dot{\theta}$ around the axis of rotation $\mathbf{u}$ as:

$$
\mathbf{u} \dot{\theta}=\mathbf{\Omega}
$$

The control of the camera orientation is thus decoupled from the position one since the former is directly available from the obtained partial pose. The position of the camera can be controlled in the image space and in the Cartesian space at the same time. In order to maintain the target in the camera field of view, we introduced in [9] the use of two independent visual features, such as the image coordinates of a target point, and of the ratio $r=\frac{d}{d^{*}}$, computed from equation (4), which controls the depth between the camera and the target. However, it is possible to design a more elegant control vector, from which stability analysis is simpler.

Consider a point $\mathbf{P}$ lying in the reference plane $\pi$. It is well known that the time derivatives of its coordinates, expressed in the current camera frame, can be written as:

$$
\dot{\mathbf{P}}=-\mathbf{V}+[\mathbf{P}]_{\times} \boldsymbol{\Omega}
$$

where $[\mathbf{P}]_{\times}$is the skew-symmetric matrix associated to vector $\mathbf{P}$. $\mathbf{V}$ and $\boldsymbol{\Omega}$ define the camera velocity screw
$\mathbf{T}=\left[\begin{array}{ll}\mathbf{V}^{T} & \boldsymbol{\Omega}^{T}\end{array}\right]^{T}$. With a perspective projection model, $\mathbf{P}$ projects onto the image plane with normalized coordinates $\mathbf{p}=\left[\begin{array}{ccc}x & y & 1\end{array}\right]^{T}=\left[\begin{array}{ccc}\frac{X}{Z} & \frac{Y}{Z} & 1\end{array}\right]^{T}$. Let us define the extended image coordinates $\mathbf{p}_{e}$ as:

$$
\mathbf{p}_{e}=\left[\begin{array}{lll}
x & y & z
\end{array}\right]^{T}=\left[\begin{array}{lll}
\frac{X}{Z} & \frac{Y}{Z} & \log (\rho)
\end{array}\right]^{T}
$$

where $z$ is a supplementary normalized coordinate which can be computed from equation (5). Then, the time variation of the extended coordinates can be written as:

$$
\dot{\mathbf{p}}_{e}=\frac{1}{Z}\left[\begin{array}{ccc}
1 & 0 & -\frac{X}{Z} \\
0 & 1 & -\frac{Y}{Z} \\
0 & 0 & 1
\end{array}\right]\left[\begin{array}{c}
\dot{X} \\
\dot{Y} \\
\dot{Z}
\end{array}\right]=-\frac{1}{d^{*} \rho} \mathbf{L}_{v}(\mathbf{p}) \dot{\mathbf{P}}
$$

where:

$$
\mathbf{L}_{v}(\mathbf{p})=\left[\begin{array}{ccc}
-1 & 0 & x \\
0 & -1 & y \\
0 & 0 & -1
\end{array}\right]
$$

Finally, the interaction matrix related to the extended image coordinates can be easily obtained:

$$
\dot{\mathbf{p}}_{e}=\left[\begin{array}{cc}
\frac{1}{d^{*} \rho} \mathbf{L}_{v} & \mathbf{L}_{\omega}
\end{array}\right] \mathbf{T}
$$

where:

$\mathbf{L}_{\omega}\left(\mathbf{p}_{e}\right)=\mathbf{L}_{v}(\mathbf{p})[\mathbf{p}]_{\times}=\left[\begin{array}{ccc}x y & -\left(1+x^{2}\right) & y \\ \left(1+y^{2}\right) & -x y & -x \\ -y & x & 0\end{array}\right]$

Equations (12) and (17) can be regrouped as follows:

$$
\left[\begin{array}{c}
\dot{\mathbf{p}}_{e} \\
\mathbf{u} \dot{\theta}
\end{array}\right]=\left[\begin{array}{cc}
\frac{1}{d^{*} \rho} \mathbf{L}_{v} & \mathbf{L}_{\omega} \\
\mathbf{0} & \mathbf{I}_{3}
\end{array}\right]\left[\begin{array}{l}
\mathbf{V} \\
\mathbf{\Omega}
\end{array}\right]=\mathbf{L}\left(d^{*}, \mathbf{p}_{e}\right) \mathbf{T}
$$

where $\mathbf{L}$ is an upper triangular matrix which is always full rank. This particular form will allow us to design a control scheme with very interesting properties.

\section{Control law}

The positioning task controlling the 6 camera d.o.f. can be described as the regulation to zero of a task function [4]. In our case:

$$
\mathbf{e}=\left[\begin{array}{ll}
\mathbf{p}_{e}-\mathbf{p}_{e}^{*} & \mathbf{u}^{T} \theta
\end{array}\right]^{T}
$$

where $\mathbf{p}_{e}^{*}$ can be obtained from the desired image acquired during the off-line learning step.

The exponential convergence of $\mathbf{p}_{e}$ toward $\mathbf{p}_{e}^{*}$ and $\mathbf{u} \theta$ toward 0 can be obtained by imposing $\dot{\mathbf{e}}=-\lambda \mathbf{e}$ (where $\lambda$ tunes the convergence rate). Assuming the target motionless (see $[2,1,10]$ otherwise), the control law is given by:

$$
\mathbf{T}=-\lambda \mathbf{L}^{-1} \mathbf{e}
$$


where $\mathbf{T}$ is the camera velocity screw sent to the robot controller. As usual, the camera velocity is considered as the control vector. In practice, small perturbations in the robot Jacobian and calibration errors in the hand to eye rigid transformation will be compensated by the closed-loop scheme. More precisely, from (19) we have:

$$
\left[\begin{array}{l}
\mathbf{V} \\
\mathbf{\Omega}
\end{array}\right]=-\lambda\left[\begin{array}{cc}
d^{*} \rho \mathbf{L}_{v}^{-1} & -d^{*} \rho \mathbf{L}_{v}^{-1} \mathbf{L}_{\omega} \\
\mathbf{0} & \mathbf{I}_{3}
\end{array}\right] \mathbf{e}
$$

Let us point out that the camera translational velocity is proportional to the desired distance $d^{*}$ between $\mathcal{F}^{*}$ and $\pi$. An approximate value has thus to be chosen during the off-line learning stage. This value has not to be precisely determined (by hand in the experiments). It will be proven, in the following section, that very large errors do not influence the stability of the system.

Let us finally note that the rotational control loop is decoupled from the translational one. A such decoupled system and the particular form of $\mathbf{L}$ allow us to obtain the convergence in the half space in front of $\pi$ if exact model and perfect measurements are assumed. However, a more realistic approach consists in generalizing the previous control as:

$$
\left[\begin{array}{l}
\mathbf{V} \\
\mathbf{\Omega}
\end{array}\right]=-\lambda \widehat{\mathbf{L}} \widehat{\mathbf{e}}=-\lambda\left[\begin{array}{cc}
\widehat{d^{*}} \widehat{\rho} \widehat{\mathbf{L}}_{v}^{-1} & -\widehat{d}^{*} \widehat{\rho} \widehat{\mathbf{L}}_{v}^{-1} \widehat{\mathbf{L}}_{\omega} \\
\mathbf{0} & \mathbf{I}_{3}
\end{array}\right] \widehat{\mathbf{e}}
$$

where hats indicate that approximations are used since the true terms are not perfectly known. The closedloop system will then be stable if $\mathbf{L} \widehat{\mathbf{L}}^{-1}$ is positive [4]. In the next section, after the demonstration of the local asymptotical stability, sufficient conditions to ensure the positiveness of the matrix $\mathbf{L} \widehat{\mathbf{L}}^{-1}$ are given.

\section{$5 \quad$ Stability analysis}

Let us assume that the only possible errors are on the intrinsic camera parameters and on $d^{*}$. The task function can be reconstructed as:

$$
\widehat{\mathbf{e}}=\left[\begin{array}{cc}
\mathbf{E}_{\tau} & 0 \\
0 & \mathbf{E}_{\theta}
\end{array}\right] \mathbf{e}
$$

with:

$$
\mathbf{E}_{\tau}=\left[\begin{array}{cc}
\delta \mathbf{A}_{11} & 0 \\
0 & 1
\end{array}\right], \quad \mathbf{E}_{\theta}=\mu\left[\begin{array}{cc}
\delta \mathbf{A}_{11} & \delta \mathbf{p}_{0} \\
0 & 1
\end{array}\right]
$$

where $\delta \mathbf{A}_{11}$ is the $(2 \times 2)$ sub-matrix of $\delta \mathbf{A}$ containing the pixel lengths (see (1)), $\delta \mathbf{p}_{0}$ is the $(2 \times 1)$ subvector containing the error on the image center and $\mu=\frac{1}{\|\delta \mathbf{A u}\|}$. The closed-loop system can be written:

$$
\dot{\mathbf{e}}=\mathbf{f}(\mathbf{e})=-\lambda \mathbf{Q}(\mathbf{e}) \mathbf{e}
$$

Noting $\nu=\frac{\widehat{d^{*}} \widehat{\rho}}{d^{*} \rho}=\frac{\widehat{d}^{*}}{d^{*}}\left\|\delta \mathbf{A}^{-T} \mathbf{n}^{*}\right\|$, matrix $\mathbf{Q}$ can be written as:

$$
\mathbf{Q}=\left[\begin{array}{cc}
\nu \mathbf{L}_{v} \widehat{\mathbf{L}}_{v}^{-1} \mathbf{E}_{\tau} & \mathbf{L}_{v}\left([\mathbf{p}]_{\times}-\nu[\widehat{\mathbf{p}}]_{\times}\right) \mathbf{E}_{\theta} \\
\mathbf{0} & \mathbf{E}_{\theta}
\end{array}\right]
$$

Function $\mathbf{f}$ is a $C^{\infty}$ vector field defined on an open subset $S$ of $S E_{3}$. It is easy to show the existence and uniqueness of the equilibrium point:

Proposition 1 The only point of equilibrium for $\mathbf{f}$, i.e a point $\mathbf{e}^{\circ} \in S$ such that $\mathbf{f}\left(\mathbf{e}^{\circ}\right)=0$, is $\mathbf{e}^{\circ}=0$.

Proof of proposition 1 The existence of the equilibrium point is evident since if $\mathbf{e}=0$, then $\mathbf{f}(\mathbf{e})=$ $\mathbf{Q}(\mathbf{e}) \mathbf{e}=0$. This equilibrium point is unique if and only if $\operatorname{det}(\mathbf{Q}(\mathbf{e})) \neq 0, \forall \mathbf{e} \in S$. Since matrix $\mathbf{Q}$ is upper triangular, its determinant can be easily calculated:

$$
\begin{aligned}
\operatorname{det}(\mathbf{Q}) & =\nu^{3} \operatorname{det}\left(\mathbf{L}_{v}\right) \operatorname{det}\left(\widehat{\mathbf{L}}_{v}^{-1}\right) \operatorname{det}\left(\mathbf{E}_{\tau}\right) \operatorname{det}\left(\mathbf{E}_{\theta}\right)= \\
& =\nu^{3} \mu^{3} \frac{\alpha_{u}^{2}}{\widehat{\alpha}_{u}^{2}} \frac{\alpha_{v}^{2}}{\widehat{\alpha}_{v}^{2}}
\end{aligned}
$$

then $\operatorname{det}(\mathbf{Q}) \neq 0, \forall \mathbf{e} \in S$ since $\nu \neq 0$ and $\mu \neq 0$.

Therefore, the task has no singularity and, if the task function decreases, it decreases towards 0 .

Theorem 1 (Local asymptotic stability) The differential system (26) is locally asymptotically stable around the equilibrium point $\mathbf{e}^{\circ}$ if and only if:

$$
\frac{\widehat{\alpha}_{u}}{\alpha_{u}}>0, \quad \frac{\widehat{\alpha}_{v}}{\alpha_{v}}>0 \quad \text { and } \quad \frac{\widehat{d}^{*}}{d^{*}}>0
$$

In practice, these conditions are easily verified.

Proof of theorem 1 Consider now the first order Taylor expansion of the non-linear differential system (26) around the equilibrium point:

$$
\dot{\mathbf{e}} \approx-\lambda \mathbf{Q}\left(\mathbf{e}^{\circ}\right)\left(\mathbf{e}-\mathbf{e}^{\circ}\right)
$$

The linearized system (30) is asymptotically stable if and only if the eigenvalues of $\mathbf{Q}\left(\mathbf{e}^{\circ}\right)$ are positive. They are given by:

$$
\begin{array}{lll}
\lambda_{1}=\nu \alpha_{u} / \widehat{\alpha}_{u}, & \lambda_{3}=\nu, & \lambda_{5}=\mu \alpha_{u} / \widehat{\alpha}_{u}, \\
\lambda_{2}=\nu \alpha_{v} / \widehat{\alpha}_{v}, & \lambda_{4}=\mu, & \lambda_{6}=\mu \alpha_{v} / \widehat{\alpha}_{v}
\end{array}
$$

Then they are positive if and only if conditions (29) are verified. Since the linearized system is asymptotically stable, the local asymptotic stability, around the equilibrium point, of the non-linear system is proven.

We now present sufficient conditions to ensure the global asymptotic stability. 
Theorem 2 (Global asymptotic stability) The differential system (26) is globally asymptotically stable, only if conditions (29) are verified, and if:

$$
\mu(\nu\|\mathbf{I}-\delta \mathbf{A}\|+|1-\nu|)^{2}\|\delta \mathbf{A}\|^{2} g^{2}(\bar{\gamma})<\nu \sigma_{1} \sigma_{2}
$$

where $\gamma=\sqrt{x^{2}+y^{2}}=\tan (\psi)$ is the tangent of the vision angle $\psi$ ( $\bar{\gamma}$ is its maximum value), and:

$$
\begin{gathered}
g^{2}(\bar{\gamma})=\frac{2+\bar{\gamma}^{2}+\sqrt{\bar{\gamma}^{2}\left(\bar{\gamma}^{2}+4\right)}}{2}\left(1+\bar{\gamma}^{2}\right) \\
\sigma=\frac{\alpha_{u}}{\widehat{\alpha}_{u}}+\frac{\alpha_{v}}{\widehat{\alpha}_{v}}-\sqrt{\left(\frac{\alpha_{u}}{\widehat{\alpha}_{u}}-\frac{\alpha_{v}}{\widehat{\alpha}_{v}}\right)^{2}+\left(\frac{\alpha_{u v}}{\widehat{\alpha}_{u}}-\frac{\widehat{\alpha}_{u v}}{\widehat{\alpha}_{u}} \frac{\alpha_{v}}{\widehat{\alpha}_{v}}\right)^{2}}>0 \\
\sigma_{1}=\sigma+1-\sqrt{(\sigma-1)^{2}+\|\mathbf{I}-\delta \mathbf{A}\|^{2}\left(1+\bar{\gamma}^{2}\right)}>0 \\
\sigma_{2}=\sigma+1-\sqrt{(\sigma-1)^{2}+\left\|\delta \mathbf{p}_{0}\right\|^{2}}>0
\end{gathered}
$$

The proof of the theorem is given in [8]. This sufficient condition depends on many parameters and has to be used with some knowledge of the system geometry. As an example, we can use condition (32) to obtain a sufficient condition when the hypothesis of exact measurements cannot be applied to the distance $d^{*}$ since it is estimated by hand. In that case, where perfect camera calibration is assumed $\left(\delta \mathbf{A}=\mathbf{I}, \sigma=\sigma_{1}=\sigma_{2}=2\right.$, $\mu=1$ and $\left.\nu=\frac{\widehat{d^{*}}}{d^{*}}\right)$, condition (32) can be written :

$$
\left(1-\frac{\widehat{d}^{*}}{d^{*}}\right)^{2} g^{2}(\bar{\gamma})<4 \frac{\widehat{d}^{*}}{d^{*}}
$$

The solution of this inequality is:

$$
1+2 \frac{1-\sqrt{g^{2}(\bar{\gamma})+1}}{g^{2}(\bar{\gamma})}<\frac{\widehat{d}^{*}}{d^{*}}<1+2 \frac{1+\sqrt{g^{2}(\bar{\gamma})+1}}{g^{2}(\bar{\gamma})}
$$

The two bounds are plotted in Figure 2 versus $\bar{\gamma}$.

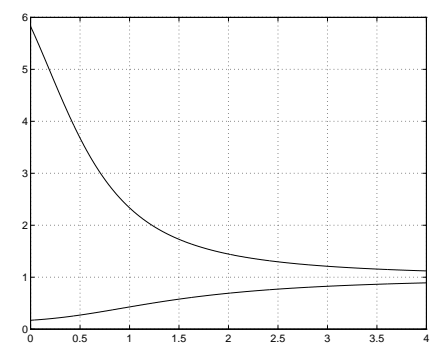

Figure 2: Stability bounds for the estimated depth

This means that, if we consider for example a camera with a $20^{\circ}$ vision angle (then $\bar{\gamma}=0.364$ ), the stability condition is verified if $0.24<\widehat{d}^{*} / d^{*}<4.22$. If the real distance $d^{*}$ is $50 \mathrm{~cm}$, the system will asymptotically converge for any initial position in the task space if $\widehat{d}^{*}$ is chosen between 12 and $211 \mathrm{~cm}$. This result definitively validates the robustness of our control scheme. Moreover, similar results can be obtained by considering camera calibration errors. Let us finally note that condition (32) is only sufficient, then the convergence can be realized even for larger errors. In the next section, we will see experimentally that the $2 \mathrm{D} 1 / 2$ visual servoing is robust also in presence of errors in the robot Jacobian.

\section{Experimental results}

The control law has been tested on a seven d.o.f. industrial robot MITSUBISHI PA10 (at EDF DER Chatou) and a six d.o.f. Cartesian robot AFMA (at IRISA). The camera is mounted on the robot endeffector. In the presented experiments, $d^{*}$ is set to $50 \mathrm{~cm}$ (while its real value is $60 \mathrm{~cm}$ ) and $\lambda=0.1$ (a faster convergence can be obtained by increasing $\lambda$ when the error becomes small). As far as calibration is concerned, two different set of parameters are used:

- coarse calibration: the pixel and focal lengths given by the camera constructor are used. The image center has been used for the optical axis projection. The transformation matrix between the camera and the robot end-effector frames is set with an accuracy to within $1 \mathrm{~cm}$ for translation and $5 \mathrm{dg}$ for rotation.

- bad calibration: a supplementary error on the camera intrinsic parameters (20\%) is added, as well as on the translation ( $5 \mathrm{~cm}$ on each axis) and on the rotation ( $5 \mathrm{dg}$ on each axis) of the transformation matrix between the camera and the robot end-effector.

The images corresponding to the desired and initial camera poses are given in Figure $3 \mathrm{a}$ and $3 \mathrm{~b}$ respectively. The target is composed by twelve white marks lying on three different planes. The extracted visual features are the image coordinates of the center of gravity of each mark. With such simple images, the control loop can be realized at video rate.

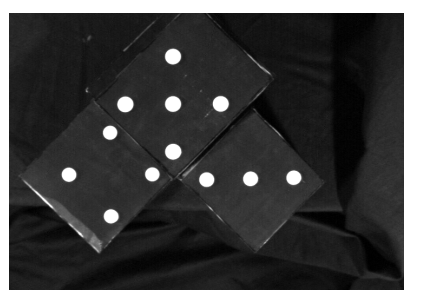

(a)

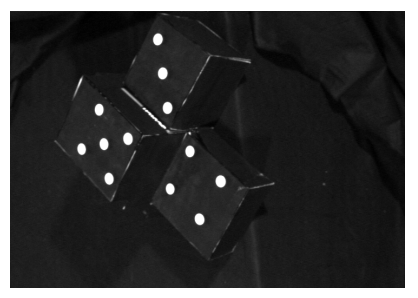

(b)
Figure 3: Images of the target for desired (a) and initial (b) camera poses 
The camera rotation (in degrees) and the direction of translation, computed from the estimated homography using the initial and desired images, are given in Table 1 in function of the camera calibration.

\begin{tabular}{|c|ccc|ccc|}
\hline calibration & $r_{x}^{\circ}$ & $r_{y}^{\circ}$ & $r_{z}^{\circ}$ & $\frac{t_{x}}{\|\mathbf{t}\|}$ & $\frac{t_{y}}{\|\mathbf{t}\|}$ & $\frac{t_{z}}{\|\mathbf{t}\|}$ \\
\hline exact & 28.1 & -33.8 & 96.1 & -0.11 & 0.99 & 0.10 \\
\hline coarse & 33.0 & -32.8 & 96.3 & -0.20 & 0.97 & 0.13 \\
\hline bad & 26.9 & -26.6 & 99.0 & -0.25 & 0.96 & 0.04 \\
\hline
\end{tabular}

Table 1: Motion parameters

The pose estimations, obtained using coarse and bad camera calibration, are not very different (compare Figure 4(b) and Figure 6(b)). For example, the maximal rotational error is around $5 \mathrm{dg}$ for coarse calibration and around $7 \mathrm{dg}$ for bad calibration. However, the resulting features points trajectories in the images (see Figure 5(b) and Figure 7(b)) show the influence of bad calibration on the convergence of the system.

\subsection{Coarse calibration}

The error on the extended image coordinates of the reference point are plotted in Figure 4a. The estimated rotation is plotted in Figure $4 \mathrm{~b}$.

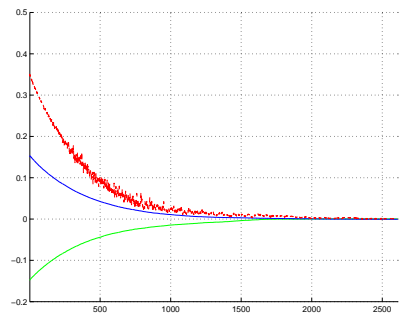

(a)

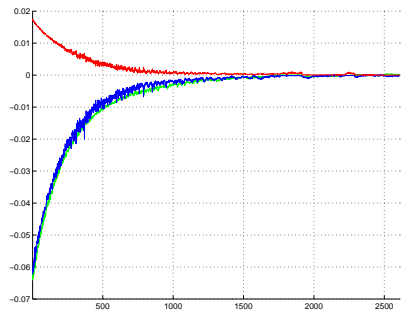

(c)

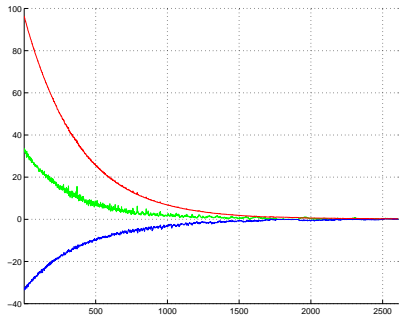

(b)

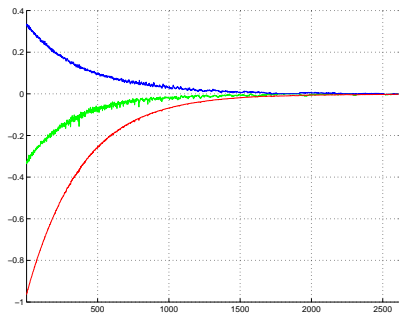

(d)
Figure 4: Error on extended image coordinates (a), $\mathbf{u} \theta$ (dg) (b), translational velocity $\mathbf{V}(\mathrm{cm} / \mathrm{s})$ (c) and rotational velocity $\boldsymbol{\Omega}(\mathrm{dg} / \mathrm{s})(\mathrm{d})$ versus iteration number
The outputs of the control law are given in Figure 4c and Figure $4 \mathrm{~d}$. The obtained results are particularly stable and robust and the error decreases exponentially to 0 . Finally, the error on the image coordinates of each target point is given in Figure 5a and the corresponding trajectory in the image is given in Figure $5 \mathrm{~b}$. The trajectory of the chosen reference point can be easily identified since it looks like a straight line in the image.

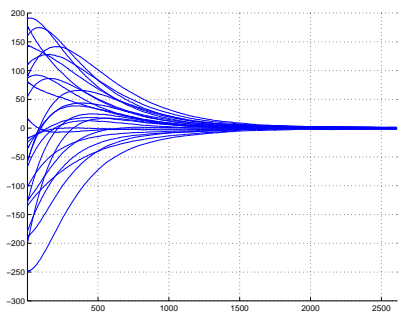

(a) (b)

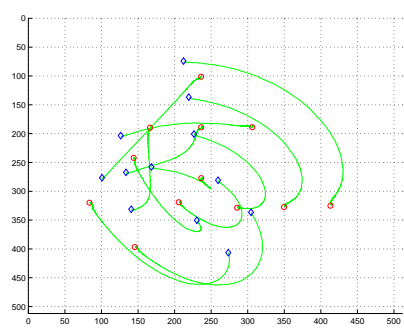

Figure 5: Error on pixels coordinates (a) and trajectory in the image (b) of the target points

The convergence of the coordinates to their desired values demonstrates the correct realization of the positioning task. We can finally note that, due to the important displacement between the initial and desired camera poses, image-based and position-based visual servoings fail in that case.

\subsection{Bad calibration}

The obtained results are given in Figure 6 and Figure 7.

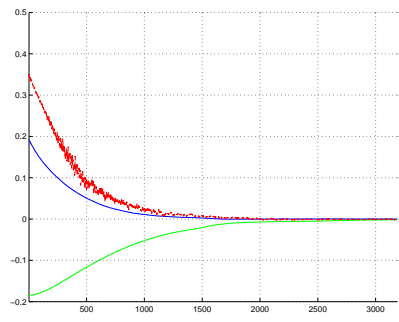

(a)

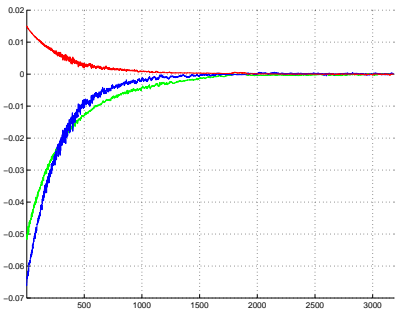

(c)

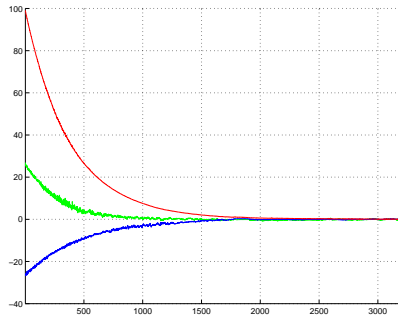

(b)

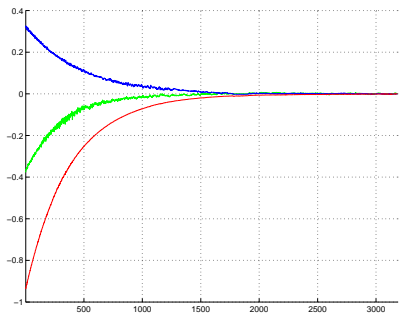

(d)
Figure 6: Error on extended image coordinates (a), $\mathbf{u} \theta$ (dg) (b), translational velocity $\mathbf{V}(\mathrm{cm} / \mathrm{s})$ (c) and rotational velocity $\boldsymbol{\Omega}(\mathrm{dg} / \mathrm{s})(\mathrm{d})$ versus iteration number 
As can be seen in Figure 6a, the convergence of the error is no more perfectly exponential. This is due to the bad calibration of the camera and the rough approximation of $d^{*}$ (which has no influence using a coarse calibration). However, even in this worse case, we can note the stability and the robustness of the control law. Contrarily to the previous experiment, the trajectory of the reference point in the image is no more a straight line since the camera is bad calibrated as well as the homogeneous transformation matrix between the camera and the robot end-effector frame. However, the convergence of the coordinates to their desired values demonstrates the correct realization of the task.

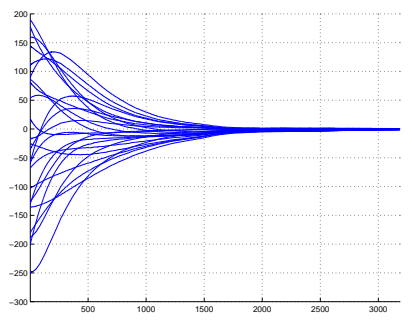

(a)

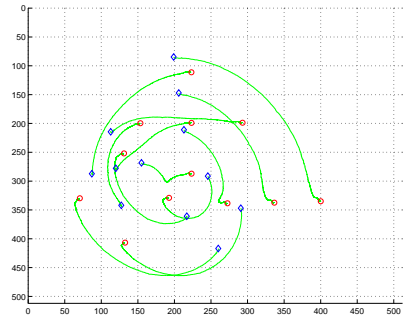

(b)
Figure 7: Error on pixels coordinates (a) and trajectory in the image (b) of the target points

\section{Conclusion}

In this paper we have proposed a new approach to vision-based robot control which presents many advantages with respect to the classical position-based and image-based visual servoings. Thanks to its simple structure analytical results on its robustness can be obtained. The necessary and sufficient conditions for local asymptotic stability and sufficient conditions for global asymptotic stability in presence of camera calibration errors have been obtained. Experimental results show the validity of our approach and its robustness not only with respect to camera calibration errors but also to robot calibration errors. Future work will be devoted to find an adaptive control scheme in order to maintain the target in the image even in presence of very large calibration errors, and to the coupling of our control scheme with real objects and complex images.

\section{Acknowledgments}

This work was supported by the national French Company of Electricity Power: EDF. We are grateful to the team manager and the researchers of the Teleoperation/Robotics group, at DER Chatou, for their participation and help.

\section{References}

[1] P. K. Allen, A. Timcenko, Y. B., and P. Michelman. Automated tracking and grasping of a moving object with a robotic hand-eye system. IEEE Trans. on Robotics and Automation, 9(2):152-165, April 1993.

[2] F. Bensalah and F. Chaumette. Compensation of abrupt motion changes in target tracking by visual servoing. In IEEE/RSJ Int. Conf. on Intelligent Robots and Systems, IROS'95, volume 1, pages 181187, Pittsburgh, Pennsylvania, August 1995.

[3] B. Espiau. Effect of camera calibration errors on visual servoing in robotics. In 3rd International Symposium on Experimental Robotics, Kyoto, Japan, October 1993.

[4] B. Espiau, F. Chaumette, and P. Rives. A new approach to visual servoing in robotics. IEEE Trans. on Robotics and Automation, 8(3):313-326, June 1992.

[5] O. Faugeras and F. Lustman. Motion and structure from motion in a piecewise planar environment. International Journal of Pattern Recognition and Artificial Intelligence, 2(3):485-508, 1988.

[6] K. Hashimoto, editor. Visual Servoing: Real Time Control of Robot manipulators based on visual sensory feedback, volume 7 of World Scientific Series in Robotics and Automated Systems. World Scientific Press, Singapore, 1993.

[7] S. Hutchinson, G. D. Hager, and P. I. Corke. A tutorial on visual servo control. IEEE Trans. on Robotics and Automation, 12(5):651-670, October 1996.

[8] E. Malis, F. Chaumette, and S. Boudet. 2D $1 / 2$ visual servoing. Technical Report 3387, INRIA, March 1998. Available on ftp://ftp.inria.fr/INRIA/publication/RR-3387.ps.gz.

[9] E. Malis, F. Chaumette, and S. Boudet. Positioning a coarse-calibrated camera with respect to an unknown object by $2 \mathrm{D} 1 / 2$ visual servoing. In IEEE International Conference on Robotics and Automation, volume 2, pages 1352-1359, Luvein, Belgium, May 1998.

[10] N. P. Papanikolopoulos, P. K. Kosla, and T. Kanade. Visual tracking of a moving target by a camera mounted on a robot: a combination of control and vision. IEEE Trans. on Robotics and Automation, 9(1):14-35, February 1993.

[11] W. J. Wilson, C. C. W. Hulls, and G. S. Bell. Relative end-effector control using cartesian position-based visual servoing. IEEE Trans. on Robotics and Automation, 12(5):684-696, October 1996. 